\title{
p21 protects cardiomyocytes against ischemia-reperfusion injury by inhibiting oxidative stress
}

\author{
HONG LI ${ }^{1 *}$, TONG ZOU ${ }^{2 *}$, SHUAI MENG $^{1}$, YUN-ZHU PENG $^{3}$ and JIE-FU YANG ${ }^{2}$ \\ ${ }^{1}$ Department of Emergency Medicine, Beijing Anzhen Hospital, Capital Medical University, Beijing 100029; \\ ${ }^{2}$ Department of Cardiology, Beijing Hospital, Beijing $100730 ;{ }^{3}$ Department of Cardiology, The First Affiliated Hospital, \\ Kunming Medical University, Kunming, Yunnan 650032, P.R. China
}

Received February 3, 2016; Accepted January 27, 2017

DOI: $10.3892 / \mathrm{mmr} .2018 .8382$

\begin{abstract}
Ischemic heart disease is a major health threat, resulting in a large number of mortalities annually worldwide. Oxidative stress is one of the main causes of cell death during ischemia-reperfusion (IR) injury. Cyclin dependent kinase inhibitor 1A (known as p21) is important in protecting tissues against IR injury, however the mechanism remains unknown. In the present study, oxygen-glucose deprivation and subsequent reoxygenation (OGD/R) in $\mathrm{H} 9 \mathrm{c} 2$ heart-derived myocytes was used as a model to study myocardial IR injury in vitro. mRNA and protein expression levels were determined by reverse transcription-quantitative polymerase chain reaction and western blotting, respectively. The levels of reactive oxygen species were measured using the fluorescence dye 2',7'-dichlorodihydrofluorescein diacetate. The present data demonstrated that $\mathrm{p} 21$ expression was upregulated by tumor protein p53 (p53) in $\mathrm{H} 9 \mathrm{c} 2$ cells exposed to OGD/R. p21 protected H9c2 cells against OGD/R-induced oxidative stress. In addition, p21 mediated upregulation of NF-E2-related factor-2 (Nrf2), a regulator of antioxidant responses, which in turn suppressed cell death in H9c2 cells subjected to OGD/R. Thus, activation of the p53/p21/Nrf2 signaling pathway may be an important adaptive response that limits oxidative injury during IR.
\end{abstract}

Correspondence to: Professor Yun-Zhu Peng, Department of Cardiology, The First Affiliated Hospital, Kunming Medical University, 295 Xichang Road, Kunming, Yunnan 650032, P.R. China

E-mail: pengyunzhu0308@163.com

Professor Jie-Fu Yang, Department of Cardiology, Beijing Hospital, 1 Dongdang Dahua Road, Beijing 100730, P.R. China

E-mail: 1taoy@263.net

*Contributed equally

Key words: ischemia-reperfusion injury, cyclin dependent kinase inhibitor 1A, oxidative stress, cardiomyocyte, NF-E2-related factor-2, tumor protein $\mathrm{p} 53$

\section{Introduction}

Ischemia-reperfusion (IR) injury occurs when blood flow to an organ is disrupted and subsequently re-introduced, and it is associated with a variety of diseases, including myocardial infarction, shock liver, and acute ischemic renal failure (1-3). Ischemic and reperfusion conditions lead to ATP depletion, accumulation of toxic metabolites, such as reactive oxygen species (ROS), and initiation of apoptotic and necrotic cascades in the affected organs $(4,5)$. The molecular mechanism by which cells and tissues adapt to IR involves the upregulation of cytoprotective genes that maintain cellular homeostasis. For instance, expression of the anti-apoptotic protein Bax inhibitor-1 (BI-1) is induced during IR injury, and bi-1-/- mice exhibit increased sensitivity to renal IR injury, suggesting that bi-1 gene induction is needed to protect tissues (5).

Cyclin dependent kinase inhibitor 1A (termed p21) is important in cell cycle regulation (6). Previously, Megyesi et al (7) have demonstrated that expression of p21 is significantly upregulated following renal ischemia. In p21-/mice, renal function is more impaired and overall mortality is significantly increased compared with wild-type mice following renal ischemia, suggesting a protective role of p21 in ischemic acute renal failure. p21 has also been demonstrated to be involved in recovery from hepatic IR injury (8). However, the molecular mechanisms by which $\mathrm{p} 21$ exerts its protective effect on IR injury are not fully understood.

Oxidative stress is a major causes of cell death during IR injury (1). p21 protects cells against oxidative stress by upregulating the ER-resident chaperone heat shock protein family A (HSPA5, also known as BIP and GRP78) and suppressing endoplasmic reticulum stress (9). Chen et al (10) reported that the antioxidant function of p21 is mediated through stabilizing NF-E2-related factor-2 (Nrf2), a transcription factor that regulates expression of various detoxification and antioxidant enzymes (11). Given the importance of oxidative stress in IR injury, the present study aimed to investigate whether $\mathrm{p} 21$ protects cardiomyocytes against IR injury by suppressing oxidative stress.

\section{Materials and methods}

Cell culture. Rat H9c2 cardiomyocytes (provided by Dr Yuanli Chen, Faculty of Basic Medicine, Kunming Medical University, 
Kumming, China) were cultured in DMEM (Invitrogen; Thermo Fisher Scientific, Inc., Waltham, MA, USA) with $10 \%$ fetal bovine serum (Invitrogen; Thermo Fisher Scientific, Inc.) and maintained in a humidified, $5 \% \mathrm{CO}_{2} / 95 \%$ air incubator at $37^{\circ} \mathrm{C}$.

Oxygen-glucose deprivation/reoxygenation (OGD/R). Cells were subjected to OGD/R as previously described (12), with minor modifications. When cells reached $60-80 \%$ confluency, they were washed with PBS and cultured in glucose-free, serum-free DMEM medium in an anoxia chamber $\left(94 \% \mathrm{~N}_{2} / 5 \% \mathrm{CO}_{2} / 1 \% \mathrm{O}_{2}\right)$ for $4 \mathrm{~h}$, then cultured in high glucose $(4,500 \mathrm{mg} / \mathrm{l}) \mathrm{DMEM}$ medium containing $10 \% \mathrm{FBS}$ in a normal cell incubator $\left(95 \%\right.$ air $\left./ 5 \% \mathrm{CO}_{2}\right)$ for an additional $12 \mathrm{~h}$. To test the role of p53 in p21 expression, cells were pre-incubated with $30 \mu \mathrm{M}$ p53 inhibitor pifithrin- $\alpha$ (Sigma-Aldrich; Merck Millipore, Darmstadt, Germany) for $30 \mathrm{~min}$.

Cell viability assay. Cell survival was measured using the 3-(4,5-dimethylthiazol-2-yl)-5-(3-carboxymethoxyphenyl)-2(4-sulfophenyl)-2H-tetrazolium inner salt (MTS) assay (Promega Corporation, Madison, WI, USA). Briefly, 1x $10^{5}$ $\mathrm{H} 9 \mathrm{c} 2$ cells/well were seeded into 96 -well plates at $37^{\circ} \mathrm{C}$ overnight. Following OGD/R, $20 \mu \mathrm{l}$ MTS solution was added in each well and cells were incubated for a further $2 \mathrm{~h}$ at $37^{\circ} \mathrm{C}$. Absorbance at $490 \mathrm{~nm}$ was measured using a microplate reader (Molecular Devices, LLC, Sunnyvale, CA, USA).

Measurement of ROS. ROS levels in cells were measured using 2',7'-dichlorodihydrofluorescein diacetate $\left(\mathrm{H}_{2} \mathrm{DCFDA}\right.$; Thermo Fisher Scientific, Inc.), as per the manufacturer's instructions. Briefly, H9c2 cells were seeded at a density of $5 \times 10^{4}$ into a 96-well plate. Following OGD/R treatment, $10 \mu \mathrm{M}$ $\mathrm{H}_{2}$ DCFDA were added and cells were incubated for a further $1 \mathrm{~h}$ at $37^{\circ} \mathrm{C}$. Dichlorofluorescein fluorescence intensity was measured by using a Spectra Max M5 fluorescent microplate reader (Molecular Devices, LLC).

Reverse transcription-quantitative polymerase chain reaction $(R T-q P C R)$. Total RNA was isolated using TRIzol reagent (Thermo Fisher Scientific, Inc.). Random-primed cDNAs were generated by reverse transcription of total RNA samples with SuperScript II, as per the manufacturer's instructions (Thermo Fisher Scientific, Inc.). qPCR was performed using the SYBR Premix Ex Taq kit (Takara Biotechnology, Co., Ltd., Dalian, China) on a LightCycler 480 system (Roche Applied Science, Penzberg, Germany), as per the manufacturer's instructions. The cycle number at which the reaction crossed an arbitrarily placed threshold (CT) was determined for each gene, and the relative amount of each mRNA to mRNA of $\beta$-actin was described using the $2^{-\Delta \Delta \mathrm{Cq}}$ method (13). The primers used for qPCR were as follows: p21 forward, 5'-TCTGCTGCTCTCCCTTCCT-3' and reverse, 5'-CACCACCACCACATACCAC-3'; tumor protein p53 (p53) forward, 5'-CCCATCCTTACCATCATCACG-3' and reverse, 5'-CAGGCACAAACACGAACCT-3'; Nrf2 forward, 5'-CCCAGCACATCCAGACAGAC-3' and reverse 5'-TCCAGG GCAAGCGACTCAT-3'; NAD(P)H quinone oxidoreductase 1 (NQO1) forward, 5'-GAAGAAAGGATGGGAGGTGGT-3' and reverse, 5'-GGTGGTGATGGAAAGCAAGG-3'; heme oxygenase-1 (HO-1) forward, 5'-GCGAAACAAGCAGAA
CCCA-3' and reverse, 5'-GGCTGGTGTGTAAGGGATGG-3'; $\beta$-actin forward, 5'-GATGGTCTTGGTTTCTGTGCC-3' and reverse, 5'-TGCTGTTTCCGCCTTCTGG-3'.

Western blotting. Cells were lysed on ice for $30 \mathrm{~min}$ in lysis buffer (BioTeke Corporation, Beijing, China). The lysates were centrifuged at $14,000 \mathrm{x}$ g for $15 \mathrm{~min}$ at $4^{\circ} \mathrm{C}$, and the supernatants were collected. The protein concentration was determined by a Bradford protein assay kit (BioTeke Corporation). Total cell lysates $(30 \mu \mathrm{g})$ were separated on a $10 \%$ SDS-polyacrylamide gel. Proteins were then transferred to polyvinylidene fluoride membranes. Membranes were blocked for non-specific binding with $5 \%$ bovine serum albumin in Tris phosphate buffered saline (TPBS) at room temperature for $30 \mathrm{~min}$, following which they were incubated overnight at $4^{\circ} \mathrm{C}$ with primary antibodies. Primary antibodies were as follows: Anti- $\beta$-actin (cat. no. A2066; 1:1,000 dilution; Sigma-Aldrich; Merck Millipore), anti-p21 (cat. no. sc-817; 1:500 dilution; Santa Cruz Biotechnology, Inc. Dallas, TX, USA), anti-p53 (cat. no. sc-126; 1:1,000 dilution; Santa Cruz Biotechnology, Inc.) and anti-Nrf2 (cat. no. sc-722; 1:500 dilution; Santa Cruz Biotechnology, Inc.). Membranes were subsequently washed with TPBS three times, and incubated with horseradish peroxidase-conjugated anti-rabbit or -mouse immunoglobin G secondary antibodies [cat. nos. M21002 and H20704 respectively; 1:5,000 dilution; Abmart (Shanghai) Co., Ltd., Shanghai, China] for $1 \mathrm{~h}$ at room temperature. Following three washes with TPBS, membranes were exposed to Amersham Hyperfilm Enhanced Chemiluminescence (GE Healthcare Bio-Sciences, Pittsburgh, PA, USA). Following this, membranes were exposed to Kodak X-OMAT film (Kodak, Rochester, NY, USA) and the films developed.

RNA interference. To knockdown the expression of p21 and $\mathrm{Nrf} 2$ by RNA interference, $\mathrm{H} 9 \mathrm{c} 2$ cells were transfected at $50 \%$ confluence with $100 \mathrm{nM}$ of p21 or Nrf2-specific small interfering RNA (siRNA; GenePharma Co., Ltd., Shanghai, China) in Opti-MEM medium (Thermo Fisher Scientific, Inc.) using Lipofectamine 2000 transfection agent (Thermo Fisher Scientific, Inc.), as per the manufacturer's instructions. Gene silencing efficiency was determined by RT-qPCR and western blotting $72 \mathrm{~h}$ post-transfection. A non-specific siRNA sequence was used as a negative control. Endogenous p21 and Nrf2 mRNA and protein expression levels were significantly ablated by siRNA in H9c2 cells compared with the negative control siRNA (Fig. 1). siRNA sequences were as follows (sense strand): p21, 5'-UCCAAUUCCCCUUAACUCGGG-3'; Nrf2, 5'-UUGUUUUCCGUAUUAAGACA-3', and negative control 5'-UUCUCCGAACGUGUCACGUTT-3'.

Statistical analysis. Data were expressed as the mean \pm standard deviation. Statistical differences between groups were analyzed using one-way analysis of variance, followed by a Student-Newman-Keuls test. $\mathrm{P}<0.05$ was considered to indicate a statistically significant difference.

\section{Results}

$O G D / R$ induces cell death through ROS. In the present study, $\mathrm{OGD} / \mathrm{R}$ in $\mathrm{H} 9 \mathrm{c} 2$ heart-derived myocytes was used as an in vitro 

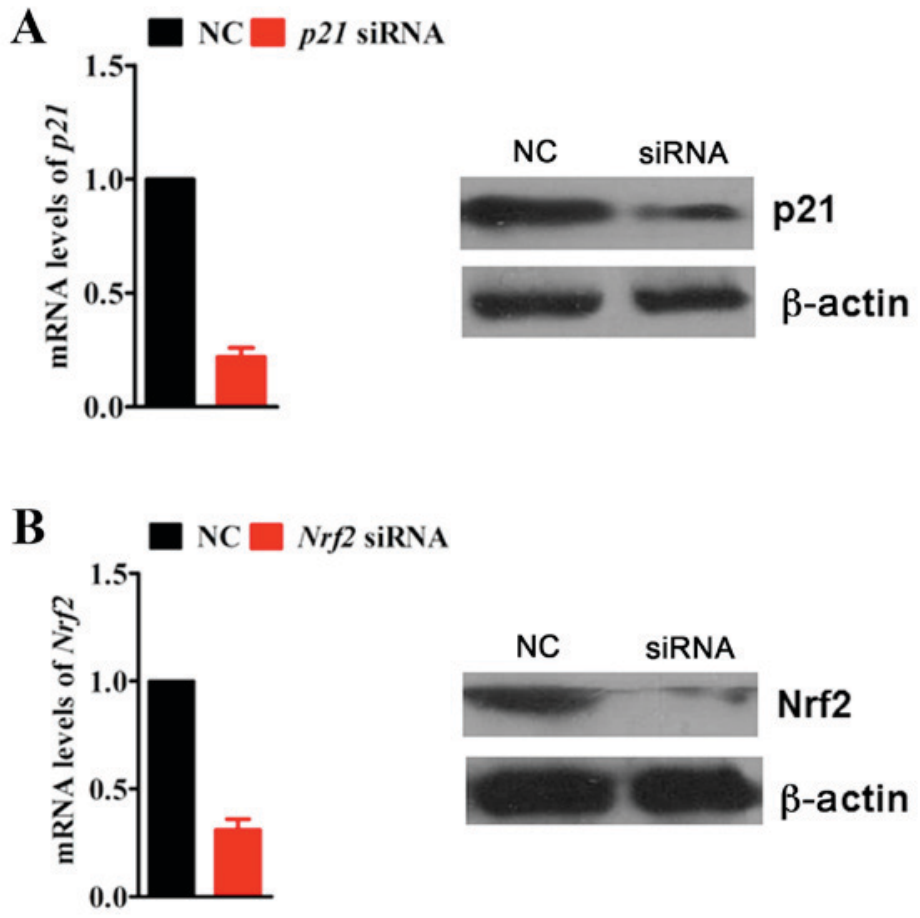

Figure 1. Knockdown of endogenous p21 and Nrf2 expression in H9c2 cells using siRNA. Cells were transfected with specific siRNAs to (A) p21 and (B) Nrf2, and efficiency of gene silencing was determined by reverse transcription-quantitative polymerase chain reaction (RT-qPCR) and western blotting (with $\beta$-actin used as a reference gene and loading control, respectively). For RT-qPCR, data are expressed as mean \pm standard deviation of three independent experiments. For western blotting, the blot is representative of three experiments. A non-specific siRNA sequence was used as negative control. NC, negative control; p21, cyclin dependent kinase inhibitor 1A; siRNA, small interfering RNA; Nrf2, NF-E2-related factor-2.

$\mathbf{A}$

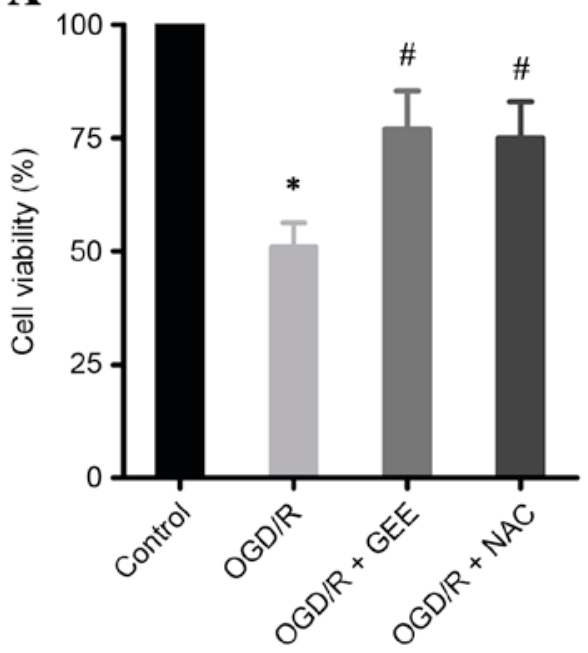

B

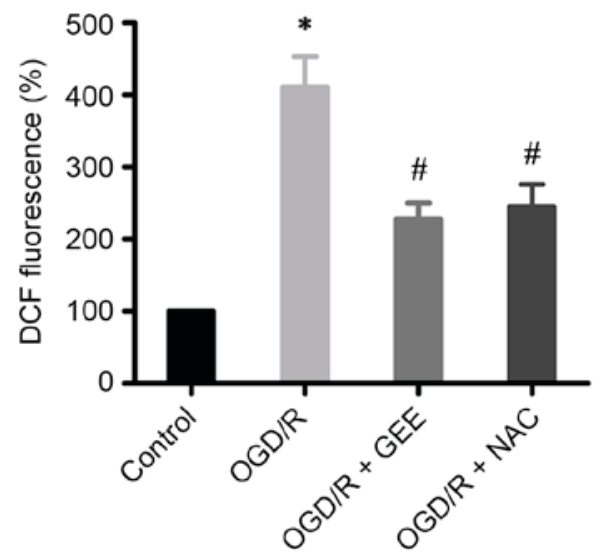

Figure 2. Oxidative stress induces cardiomyocyte cell death following OGD/R. H9c2 cells were treated with 5 mM antioxidants, GEE or NAC, for 30 min prior to being subjected to OGD/R. (A) Cell viability was assessed by MTS assay. (B) Reactive oxygen species levels were evaluated by DCF fluorescence measurements. Results are presented as the mean (\%) relative to untreated cells (control) + standard deviation of 4 experiments. ${ }^{*} \mathrm{P}<0.05$ vs. control and ${ }^{\#} \mathrm{P}<0.05$ vs. OGD/R alone. OGR/R, oxygen-glucose deprivation/reoxygenation; GEE, glutathione ethyl ester; NAC, N-acetylcysteine; MTS, 3-(4,5-dimethylthiazol-2-yl)-5-(3-carboxymethoxyphenyl)-2-(4-sulfophenyl)-2H-tetrazolium inner salt.

model to study IR. A significant increase in cell death was detected by MTS assay in OGD/R-treated H9c2 cells compared with untreated cells (Fig. 2A). Oxidative stress has been demonstrated to be important in acute myocardial infarction $(1,14)$. Therefore, ROS levels were assessed in OGD/R-treated H9c2 cells using an $\mathrm{H}_{2}$ DCF-DA probe. The results demonstrated that $\mathrm{OGD} / \mathrm{R}$ induced a 4-fold increase in ROS levels in H9c2 cells, compared with the untreated cells (Fig. 2B). To investigate whether cell death in OGD/R-treated cells was mediated by ROS, the experiment was repeated in the presence of two antioxidants, glutathione ethyl ester (GEE) and $\mathrm{N}$-acetylcysteine (NAC). Both GEE and NAC significantly reduced cell death ROS production in OGD/R-treated H9c2 cells, compared with cells that received no antioxidant treatment (Fig. 2A and B, 


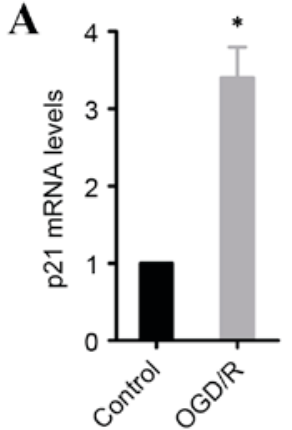

B

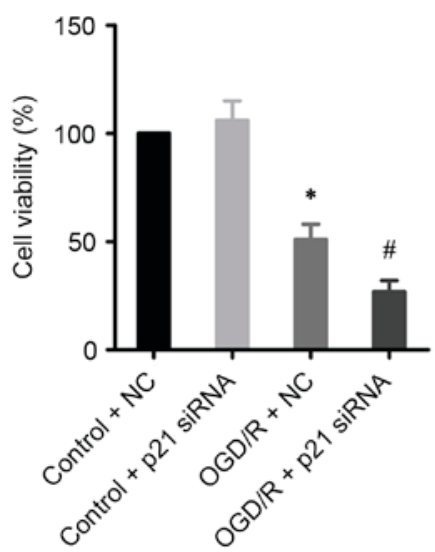

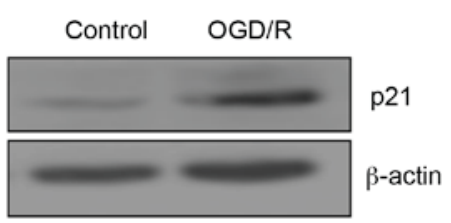

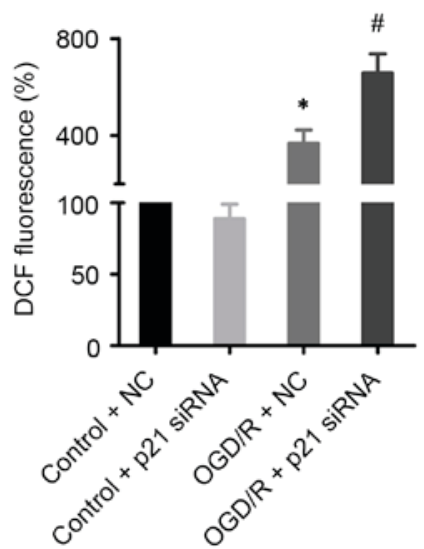

Figure 3. p21 protects cells against OGD/R-induced cell death and oxidative stress. (A) H9c2 cells were treated with OGD/R and expression of 21 was evaluated by reverse transcription-quantitative polymerase chain reaction and western blotting. Results are plotted as the mean relative to $\beta$-actin + standard deviation of 4 experiments. ${ }^{*} \mathrm{P}<0.05$ vs. control (untreated cells). (B) H9c2 cells were transfected with either p21-targeting siRNA or control siRNA, treated with OGD/R, and measured for cell viability and reactive oxygen species production. Results are plotted as \% relative to untreated, control siRNA transfected cells (mean \pm standard deviation of 4 experiments). ${ }^{*} \mathrm{P}<0.05$ vs. control $+\mathrm{NC}$ (untreated cells transfected with control siRNA) and ${ }^{\#} \mathrm{P}<0.05 \mathrm{vs}$. OGD/R $+\mathrm{NC}$ (OGD/R-treated cells transfected with control siRNA). p21, cyclin dependent kinase inhibitor 1A; OGD/R, oxygen-glucose deprivation/reoxygenation; NC, negative control; siRNA, small interfering RNA.

respectively). The present results further confirm that ROS is important in OGD/R-induced cell death in cardiomyocytes.

p21 prevents $O G D / R$-induced cell death. p21 expression is often upregulated during IR injury $(8,15)$. In the present study, mRNA and protein expression levels of p21 were demonstrated to be significantly increased in OGD/R-treated H9c2 cells, compared with untreated cells (Fig. 3A). To address whether this induction of $\mathrm{p} 21$ expression is required for cell survival, p21 expression was silenced using siRNA. The results demonstrated that knockdown of p21 significantly promoted cell death and increased ROS production in OGD/R-treated H9c2 cells, compared with OGD/R-treated cells transfected with control siRNA (Fig. 3B).

Upregulation of p21 is mediated by $p 53$. As $\mathrm{p} 21$ is a target gene of p53, whether induction of p21 in OGD/R-treated cells may be dependent on p53 was investigated. Initially, the effect of OGD/R treatment on p53 expression was examined. The results demonstrated that $\mathrm{OGD} / \mathrm{R}$ treatment in $\mathrm{H} 9 \mathrm{c} 2$ cells markedly increased the protein expression levels of p53, whereas the mRNA expression levels were not significantly different from untreated cells (Fig. 4A). Subsequently, the effect of the p53 inhibitor pifithrin- $\alpha$ (16) on p21 expression was examined. The results demonstrated that $\mathrm{p} 53$ inhibition significantly decreased the OGD/R-induced expression of p21 in $\mathrm{H} 9 \mathrm{c} 2$ cells, at the protein and the mRNA level (Fig. 4B). These data indicate that p53 is involved in the upregulation of p21 following OGD/R.

$N r f 2$ is required for survival following $O G D / R$. Transcription factor Nrf2 is a regulator of cell antioxidant responses, and it is elevated in IR injury (4). Therefore, expression of Nrf2 was examined following OGD/R treatment in $\mathrm{H} 9 \mathrm{c} 2$ cells. As demonstrated in Fig. 5A, OGD/R treatment increased Nrf2 protein expression levels, but the mRNA levels were not changed, compared with untreated cells. These data suggest that Nrf2 may be important in the cellular defense against OGD/R-induced cell death. Indeed, knockdown of Nrf2 by siRNA significantly reduced cell death and increased ROS production in OGD/R-treated H9c2 cells compared with OGD/R-treated cells transfected with control siRNA (Fig. 5B).

OGD/R induced Nrf2-regulated antioxidant gene expression via $p 21$. Previous studies have demonstrated that $\mathrm{p} 21$ may stabilize Nrf2 by inhibiting its degradation (10), raising the hypothesis that OGD/R-induced Nrf2 protein upregulation may be mediated by $\mathrm{p} 21$. To test this hypothesis, endogenous p21 expression was silenced by siRNA in H9c2 cells. Knockdown of p21 resulted in markedly reduced protein 
A

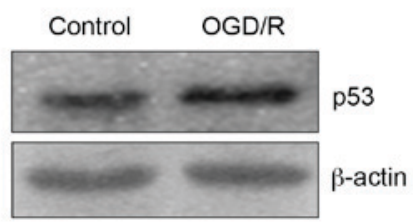

B

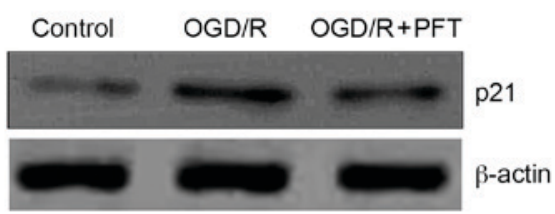

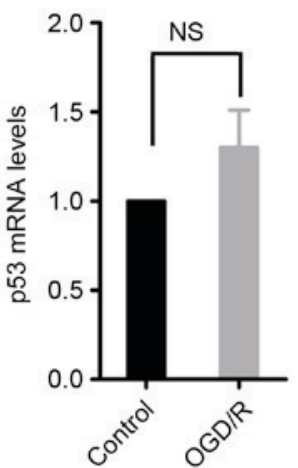

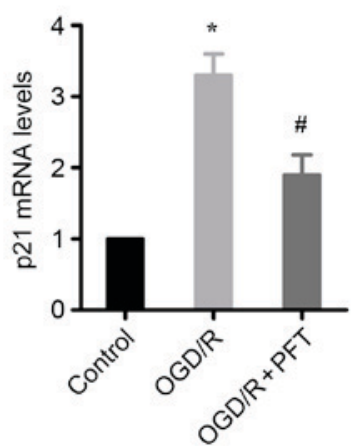

Figure 4. Upregulation of $\mathrm{p} 21$ expression is mediated by $\mathrm{p} 53$. (A) H9c2 cells were treated with OGD/R and p53 expression levels were examined by western blotting and RT-qPCR. (B) H9c2 cells were treated with a p53 specific inhibitor, PFT, and OGD/R, and p21 expression levels were examined by western blotting and RT-qPCR. Results were normalized to $\beta$-actin and plotted as the mean relative to control + standard deviation of 4 experiments. " $\mathrm{P}<0.05$ vs. control (untreated cells) and ${ }^{\#} \mathrm{P}<0.05$ vs. OGD/R alone. RT-qPCR, reverse transcription-quantitative polymerase chain reaction; OGD/R, oxygen-glucose deprivation/reoxygenation; 553 , tumor protein $\mathrm{p} 53$; NS, not significant; PFT, pifithrin- $\alpha$; 21 , cyclin dependent kinase inhibitor 1A.

A

\section{B}
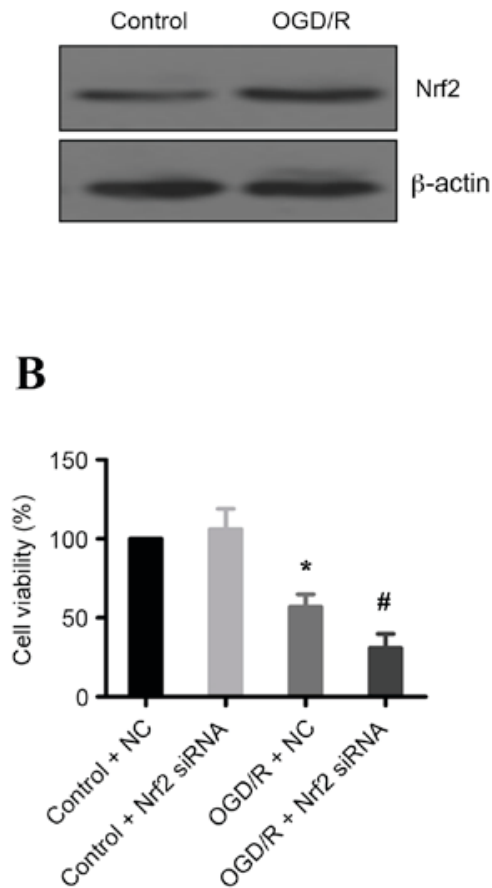
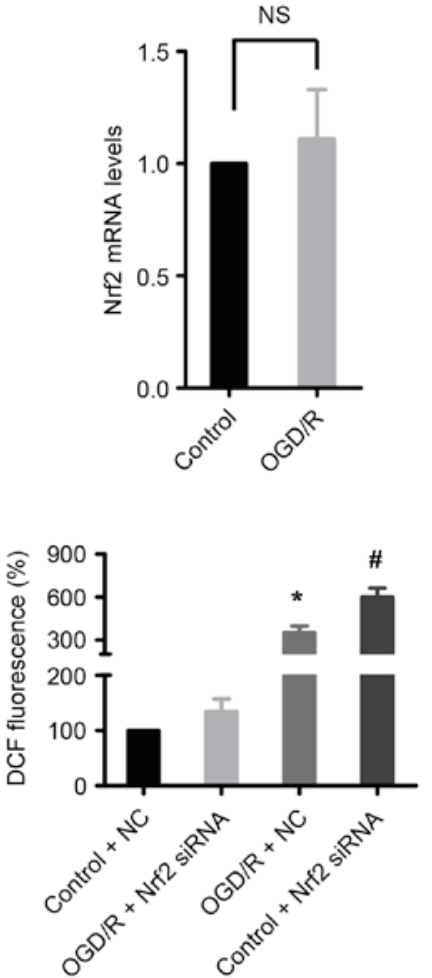

Figure 5. Nrf2 protects cells against OGD/R-induced cell death and oxidative stress. (A) H9c2 cells were treated with OGD/R and Nrf2 expression levels were examined by western blotting and reverse transcription-quantitative polymerase chain reaction. Results were normalized to $\beta$-actin. (B) $\mathrm{H} 9 \mathrm{c} 2$ cells were transfected with either Nrf2-targeting siRNA or control siRNA, treated with OGD/R, and measured for cell viability and reactive oxygen species production. Results are plotted as \% relative to untreated, control siRNA transfected cells (mean \pm standard deviation of 4 experiments). ${ }^{*} \mathrm{P}<0.05 \mathrm{vs}$. control $+\mathrm{NC}$ (untreated cells transfected with control siRNA) and ${ }^{\prime \prime} \mathrm{P}<0.05$ vs. OGD/R + NC (OGD/R-treated cells transfected with control siRNA). OGD/R, oxygen-glucose deprivation/reoxygenation; Nrf2, NF-E2-related factor-2; NS, not significant; NC, negative control; siRNA, small interfering RNA; DCF, dichlorodihydrofluorescein. 
A

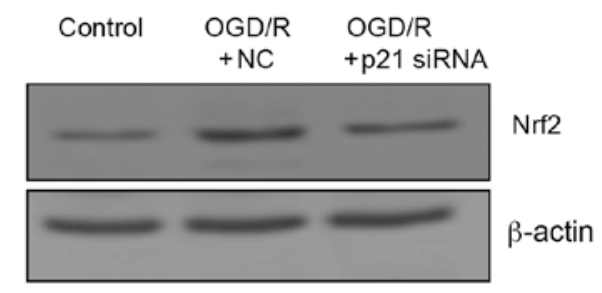

B
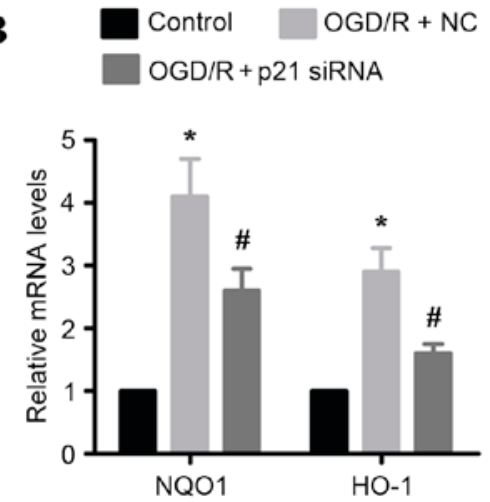

Figure 6. Upregulation of Nrf2 and antioxidant marker expression is mediated by p21. H9c2 cells were transfected with either p21-targeting siRNA or control siRNA, then treated with OGD/R. (A) Nrf2 protein expression levels were examined by western blotting. (B) NQO1 and HO-1 mRNA expression levels were examined by reverse transcription-quantitative polymerase chain reaction. Results were normalized to $\beta$-actin and plotted as the mean relative to untreated cells + standard deviation of 4 experiments. ${ }^{*} \mathrm{P}<0.05$ vs. control (untreated cells) and ${ }^{\#} \mathrm{P}<0.05$ vs. OGD/R + NC (OGD/R-treated cells transfected with control siRNA). OGD/R, oxygen-glucose deprivation/reoxygenation; NC, negative control siRNA; p21, cyclin dependent kinase inhibitor 1A; siRNA, small interfering RNA; Nrf2, NF-E2-related factor-2; NQO1, NAD(P)H quinone oxidoreductase 1; HO-1, heme oxygenase-1.

expression levels of Nrf2 in OGD/R-treated H9c2 cells compared with OGD/R-treated cells transfected with control siRNA (Fig. 6A). In addition, the mRNA expression levels of two known oxidative stress markers, and gene targets of Nrf2 (NQO1 and HO-1) were examined. As expected, the mRNA expression levels of NQO1 and HO-1 were significantly upregulated following OFD/R treatment in $\mathrm{H} 9 \mathrm{c} 2$ cells compared with untreated cells (Fig. 6B). However, this effect was significantly attenuated in p21 silenced OFD/R-treated cells, compared to OFD/R-treated cells transfected with a control siRNA (Fig. 6B). Taken together, these results indicate that p21 is important in activating the Nrf2-mediated antioxidant response following $\mathrm{OGD} / \mathrm{R}$.

\section{Discussion}

Myocardial apoptosis is critical in the pathogenesis of acute myocardial infarction and heart failure (17). Oxidative stress is one of the main causes of myocardial apoptosis following heart IR damage (14). The present study demonstrated that the p53/p21/Nrf2 pathway protects against OGD/R-induced H9c2 cell death by suppressing ROS production. Thus, induction of the $\mathrm{p} 53 / \mathrm{p} 21 / \mathrm{Nrf} 2$ pathway may represent an important adaptive cell response to limit oxidative injury during IR.

The mechanism underlying the upregulation of p21 during IR injury remains controversial. p21 expression is significantly elevated in the liver of mice that are subjected to hepatic IR injury, through activation of p53, a known regulator of p21 (8). However, Yoshida et al (15) demonstrated that the expression of activating transcription factor 3 (ATF3) is significantly increased in the kidney of a mouse model of IR injury. Overexpression of ATF3 leads to upregulation of p21 and downregulation of p53 in human kidney-2 cells subjected to oxidative stress (15), suggesting that $\mathrm{p} 21$ may be a downstream target of ATF3, rather than p53, during IR injury. In the present study, it was demonstrated that p53 inhibition suppressed the p21 expression, indicating that OGD/R-induced p21 upregulation is p53-dependent. Notably, p53 protein levels, rather than mRNA levels, were upregulated in H9c2 cells following OGD/R. Thus, post-transcriptional regulation may be a key process in the control of p53 expression. A recent study has revealed that $\mathrm{OGD} / \mathrm{R}$ significantly inhibits the phosphorylation of AKT serine/threonine kinase 1 (Akt) in H9c2 cells (18). It is well established that Akt phosphorylates the E3 ubiquitin ligase MDM2 proto-oncogene, enhancing MDM2-mediated degradation of p53 by ubiquitination (19). It is very likely therefore that inactivation of Akt may lead to accumulation of p53 protein following OGD/R, due to decreased MDM2-mediated degradation. Further studies will be required to clarify this hypothesis.

The transcription factor $\mathrm{Nrf2A}$ is a master regulator of phase-II detoxification enzymes and antioxidant genes through binding with antioxidant response elements within the promoters of these genes $(20,21)$. Previous studies have demonstrated that IR induces Nrf2 expression or upregulates Nrf2-dependent gene expression in liver and kidney $(4,22)$. Furthermore, serum urea nitrogen levels in Nrf2-null mice are higher than those in wild-type mice following IR (22). These data suggest that Nrf2 is a crucial molecule for the coordinated cytoprotective response to oxidative stress during IR. In the present study, it was demonstrated that the upregulation of Nrf2 expression by OGD/R was mediated by p21 in cardiomyocytes. Under basal conditions, an E3 ubiquitin ligase, kelch like ECH associated protein 1 (Keap1), targets Nrf2 for ubiquitination-mediated degradation (23). Notably, a previous study has demonstrated that p21 can directly interact with Nrf2 and thus competes with Keap1 for Nrf2 binding, suppressing ubiquitination of Nrf2 (10). Thus, the OGD/R-induced upregulation of Nrf2 via p21 observed in the present study, may be explained by this previously reported p21 capacity to stabilize Nrf2 and suppress its ubiquitination and degradation.

In summary, the present study demonstrated that upregulation of p21 following OGD/R was p53-dependent. p21 protected cardiomyocytes from IR injury by suppressing oxidative stress, in part through Nrf2, a master regulator of antioxidant responses. These findings provided valuable insights into the p53/p21/Nrf2 signaling in ischemic heart disease. Further in vivo investigations will be required in the future to fully comprehend the role of this pathway in IR-damaged cardiomyocytes. 


\section{Acknowledgements}

We thank Dr Yuanli Chen for kindly providing the H9c2 cells. The present study was supported in part by a grant (grant no. 81170171) from the National Natural Science Foundation of China (to JFY), and a grant (grant no. 2012FB034) from the Joint fund for Yunnan Department of Science and Technology-Kunming Medical University.

\section{References}

1. Sen CK, Khanna S and Roy S: Perceived hyperoxia: Oxygen-induced remodeling of the reoxygenated heart. Cardiovasc Res 71: 280-288, 2006.

2. Schriewer JM, Peek CB, Bass J and Schumacker PT: ROS-mediated PARP activity undermines mitochondrial function after permeability transition pore opening during myocardial ischemia-reperfusion. J Am Heart Assoc 2: e000159, 2013.

3. Eltzschig HK and Eckle T: Ischemia and reperfusion-from mechanism to translation. Nat Med 17: 1391-1401, 2011.

4. Leonard MO, Kieran NE, Howell K, Burne MJ, Varadarajan R, Dhakshinamoorthy S, Porter AG, O'Farrelly C, Rabb H and Taylor CT: Reoxygenation-specific activation of the antioxidant transcription factor Nrf2 mediates cytoprotective gene expression in ischemia-reperfusion injury. FASEB J 20: 2624-2626, 2006.

5. Bailly-Maitre B, Fondevila C, Kaldas F, Droin N, Luciano F, Ricci JE, Croxton R, Krajewska M, Zapata JM, Kupiec-Weglinski JW, et al: Cytoprotective gene bi-1 is required for intrinsic protection from endoplasmic reticulum stress and ischemia-reperfusion injury. Proc Natl Acad Sci USA 103: 2809-2814, 2006.

6. Weinberg WC and Denning MF: P21Waf1 control of epithelial cell cycle and cell fate. Crit Rev Oral Biol Med 13: 453-464, 2002.

7. Megyesi J, Andrade L, Vieira JM Jr, Safirstein RL and Price PM: Positive effect of the induction of p21WAF1/CIP1 on the course of ischemic acute renal failure. Kidney Int 60: 2164-2172, 2001.

8. Barone S, Okaya T, Rudich S, Petrovic S, Tenrani K, Wang Z, Zahedi K, Casero RA, Lentsch AB and Soleimani M: Distinct and sequential upregulation of genes regulating cell growth and cell cycle progression during hepatic ischemia-reperfusion injury. Am J Physiol Cell Physiol 289: C826-C835, 2005.

9. Vitiello PF, Wu YC, Staversky RJ and O'Reilly MA: p21 (Cip1) protects against oxidative stress by suppressing ER-dependent activation of mitochondrial death pathways. Free Radic Biol Med 46: 33-41, 2009.
10. Chen W, Sun Z, Wang XJ, Jiang T, Huang Z, Fang D and Zhang DD: Direct interaction between Nrf2 and p21 (Cip1/WAF1) upregulates the Nrf2-mediated antioxidant response. Mol Cell 34: 663-673, 2009.

11. Lau A, Villeneuve NF, Sun Z, Wong PK and Zhang DD: Dual roles of Nrf2 in cancer. Pharmacol Res 58: 262-270, 2008.

12. Wu X, He L, Chen F, He X, Cai Y, Zhang G, Yi Q, He M and Luo J: Impaired autophagy contributes to adverse cardiac remodeling in acute myocardial infarction. PLoS One 9: e112891, 2014.

13. Livak KJ and Schmittgen TD: Analysis of relative gene expression data using real-time quantitative PCR and the 2(-Delta Delta C (T))Method. Methods 25: 402-408, 2001.

14. Ide T, Tsutsui H, Kinugawa S, Utsumi H, Kang D, Hattori N, Uchida K, Arimura Ki, Egashira K and Takeshita A: Mitochondrial electron transport complex I is a potential source of oxygen free radicals in the failing myocardium. Circ Res 85: 357-363, 1999.

15. Yoshida T, Sugiura H, Mitobe M, Tsuchiya K, Shirota S, Nishimura S, Shiohira S, Ito H, Nobori K, Gullans SR, et al: ATF3 protects against renal ischemia-reperfusion injury. J Am Soc Nephrol 19: 217-224, 2008.

16. Komarov PG, Komarova EA, Kondratov RV, Christov-Tselkov K, Coon JS, Chernov MV and Gudkov AV: A chemical inhibitor of p53 that protects mice from the side effects of cancer therapy. Science 285: 1733-1737, 1999.

17. Abbate A, Bussani R, Amin MS, Vetrovec GW and Baldi A: Acute myocardial infarction and heart failure: Role of apoptosis. Int J Biochem Cell Biol 38: 1834-1840, 2006.

18. Xu F, Yu H, Liu J and Cheng L: Pyrroloquinoline quinone inhibits oxygen/glucose deprivation-induced apoptosis by activating the PI3K/AKT pathway in cardiomyocytes. Mol Cell Biochem 386: 107-115, 2014.

19. Ogawara Y,Kishishita S, Obata T, Isazawa Y, Suzuki T, Tanaka K, Masuyama N and Gotoh Y: Akt enhances Mdm2-mediated ubiquitination and degradation of p53. J Biol Chem 277: 21843-21850, 2002.

20. Jaiswal AK: Nrf2 signaling in coordinated activation of antioxidant gene expression. Free Radic Biol Med 36: 1199-1207, 2004.

21. Kobayashi M and Yamamoto M: Molecular mechanisms activating the Nrf2-Keap1 pathway of antioxidant gene regulation. Antioxid Redox Signal 7: 385-394, 2005.

22. Tanaka Y, Maher JM, Chen C and Klaassen CD: Hepatic ischemia-reperfusion induces renal heme oxygenase-1 via NF-E2-related factor 2 in rats and mice. Mol Pharmacol 71: 817-825, 2007.

23. Kobayashi A, Kang MI, Watai Y, Tong KI, Shibata T, Uchida K and Yamamoto M: Oxidative and electrophilic stresses activate Nrf2 through inhibition of ubiquitination activity of Keap1. Mol Cell Biol 26: 221-229, 2006. 